\title{
Discrete Quantitative Nodal Theorem
}

\author{
László Lovász* \\ Alfréd Rényi Institute of Mathematics \\ Budapest, Hungary \\ laszlo.lovasz@ttk.elte.hu
}

Submitted: Oct 12, 2020; Accepted: Apr 29, 2021; Published: Sep 24, 2021

(C) The author. Released under the CC BY-ND license (International 4.0).

\begin{abstract}
We prove a theorem that can be thought of as a common generalization of the Discrete Nodal Theorem and (one direction of) Cheeger's Inequality for graphs. A special case of this result will assert that if the second and third eigenvalues of the Laplacian are at least $\varepsilon$ apart, then the subgraphs induced by the positive and negative supports of the eigenvector belonging to $\lambda_{2}$ are not only connected, but edge-expanders (in a weighted sense, with expansion depending on $\varepsilon$ ).
\end{abstract}

Mathematics Subject Classifications: 05C50, 05C40

\section{Introduction}

In the theory of Riemannian manifolds, two basic theorems connect the geometry of the manifold to the spectrum of the Laplace operator on the manifold: Courant's Nodal Theorem and Cheeger's Inequality. Both of these have analogues in graph theory.

It is a basic simple fact that the graph is connected if and only if the smallest eigenvalue of its (combinatorial) Laplacian (which is always 0) has multiplicity one. Discrete Cheeger inequalities (Alon and Milman [2], Alon [1], Dodziuk and Kendall [6], Jerrum and Sinclair [12]) give a quantitative version of this: Roughly speaking, a graph is an expander if and only if the second smallest eigenvalue of its Laplacian is bounded away from zero.

The simplest version of the Discrete Nodal Theorem asserts that if $x$ is an eigenvector of the Laplacian of a connected graph $G$ belonging to the second smallest eigenvalue, and this eigenvalue has multiplicity one, then the positive and negative supports of $x$ induce connected subgraphs (Fiedler [9]). If the second smallest eigenvalue has higher multiplicity, there are exceptions (a simple example is the 3-star), but they can be characterized $[10,11]$.

*supported by ERC Synergy Grant No. 810115. 
The Discrete Nodal Theorem was extended to higher eigenvectors by Fiedler [9], Colin de Verdière [4], Davies, Gladwell, Leytold and Stadler [7], and Duval and Reiner [8]; to embedded graphs by Lin, Lippner, Mangoubi and Yau [13]; see also Bıyıkoğlu, Leydold and Stadler [3]. To motivate our results, let us quote a simple version. A nodal domain of a vector $v \in \mathbb{R}^{V}$ is a connected component of the subgraph induced by its positive support, or a connected component of the subgraph induced by its negative support.

Theorem 1. Let $G$ be a connected graph, let $\lambda_{1}=0 \leqslant \lambda_{2} \leqslant \lambda_{3} \leqslant \ldots \leqslant \lambda_{n}$ be the eigenvalues of its Laplacian, and assume that $\lambda_{k}<\lambda_{k+1}$ for some $k \geqslant 2$. Let $y$ be an eigenvector belonging to $\lambda_{k}$. Then the number of nodal domains of $y$ is at most $k$.

There are conditions other than $\lambda_{k}<\lambda_{k+1}$ to guarantee that the number of nodal domains is at most $k$, for example, that $y$ has minimal support among all eigenvectors belonging to $\lambda_{k}$.

In this note we prove a theorem that can be thought of as a common generalization of the Discrete Nodal Theorem and (one direction of) Cheeger's Inequality for graphs. A special case of our results will assert that if the second and third eigenvalues of the Laplacian are at least $\varepsilon$ apart, then the subgraphs induced by the positive and negative supports of the eigenvector belonging to $\lambda_{2}$ are not only connected, but edge-expanders (in a weighted sense, with an expansion rate depending on $\varepsilon$ ).

\section{The main result}

Let $\left(w_{i}: i \in V\right)$ be a weighting of the nodes of the graph $G=(V, E)$ with nonnegative weights. For $S \subseteq V$, set $w(S)=\sum_{i \in S} w_{i}$, and $\nabla(S)=\{i j \in E: i \in S, j \in V \backslash S\}$. If $w(S)>0$, we define the edge-expansion of $S$ as

$$
\Psi_{w}(S)=\Psi_{G, w}(S)=\frac{1}{w(S)} \sum_{i j \in \nabla(S)} \sqrt{w_{i} w_{j}} .
$$

We say that $G$ is a c-expander with respect to $w(c>0)$, if $\Psi_{w}(S) \geqslant c$ for every subset $S \subseteq V$ with $0<w(S)<w(V) / 2$.

To generalize this notion to multiway cuts, it is easier to formulate the contrapositive. We say that $(G, w)$ is $(k, c)$-partitionable, if $V$ has a partition $V=S_{1} \cup \cdots \cup S_{k}$ into sets with $w\left(S_{i}\right)>0$ such that $\Psi_{w}\left(S_{i}\right)<c$ for all $1 \leqslant i \leqslant k$. It is easy to check that if $k>2$, then merging two classes in such a partition, the new class $S_{i} \cup S_{j}$ satisfies $\Psi_{w}\left(S_{i} \cup S_{j}\right)<c$. Hence every $(k, c)$-partitionable weighted graph is $(k-1, c)$-partitionable as well.

For a vector $x \in \mathbb{R}^{V}$, we denote its positive and negative support by $\operatorname{supp}_{+}(x)=\{i \in$ $\left.V: x_{i}>0\right\}$ and $\operatorname{supp}_{-}(x)=\left\{i \in V: x_{i}<0\right\}$, and by $G_{x}^{+}$and $G_{x}^{-}$, the subgraphs of $G$ induced by $\operatorname{supp}_{+}(x)$ and $\operatorname{supp}_{-}(x)$ respectively.

Theorem 2. Let $\lambda_{1}=0 \leqslant \lambda_{2} \leqslant \lambda_{3} \leqslant \ldots \leqslant \lambda_{n}$ be the eigenvalues of the Laplacian $L$ of a graph $G=(V, E)$. Let $y$ be an eigenvector belonging to $\lambda_{k}(1 \leqslant k \leqslant n)$, and set $w_{i}=y_{i}^{2}$ and $c=\left(\lambda_{k+1}-\lambda_{k}\right) / 2$. Suppose that the weighted graph $\left(G_{y}^{+}, w\right)$ is $(a, c)$-partitionable, and $\left(G_{y}^{-}, w\right)$ is $(b, c)$-partitionable. Then $a+b \leqslant k$. 
Proof. Let us write $G^{+}=G_{y}^{+}, V^{+}=\operatorname{supp}_{+}(y)$ and $\Psi^{+}(S)=\Psi_{G^{+}, w}(S)$ for $S \subseteq V^{+}$, and define $G^{-}, V^{-}$and $\Psi^{-}$analogously. Note that $\Psi^{+}(S)=0$ if $S$ induces a connected component of $G^{+}$. Let $V^{+}=V_{1} \cup \cdots \cup V_{a}$ be a partition with $\Psi^{+}\left(V_{i}\right)<c$, and let $V^{-}=V_{a+1} \cup \cdots \cup V_{a+b}$ be an analogous partition. Let us assume (by way of contradiction) that $a+b \geqslant k+1$; we may assume (by merging partition classes) that $a+b=k+1$.

Let $M=L-\lambda_{k} I$, so that $M y=0$. Let $y^{i} \in \mathbb{R}^{V}$ denote the vector obtained from $y$ by replacing all entries in $V \backslash V_{i}$ by 0 . This vector is nonzero, because $\left|y^{i}\right|^{2}=w\left(V_{i}\right)>0$ by the definition of $(k, c)$-partitionable graphs. Furthermore, $y^{i} \geqslant 0$ for $1 \leqslant i \leqslant a$, $y^{i} \leqslant 0$ for $a+1 \leqslant i \leqslant a+b$, and $y=y^{1}+\cdots+y^{k+1}$. Let $z_{i}=\left|y^{i}\right|, \widehat{y}^{i}=\left(1 / z_{i}\right) y^{i}$, and $z=\left(z_{1}, \ldots, z_{k+1}\right)^{\top}$. Consider the $(k+1) \times(k+1)$ matrix $B$ defined by

$$
B_{i j}=\left\langle\widehat{y}^{i}, M \widehat{y}^{j}\right\rangle=\frac{1}{z_{i} z_{j}}\left\langle y^{i}, M y^{j}\right\rangle
$$

and let $\mu_{1} \leqslant \ldots \leqslant \mu_{k+1}$ be its eigenvalues. Note that for $i \leqslant a$, we have

$$
\Psi^{+}\left(V_{i}\right)=\frac{1}{z_{i}^{2}} \sum_{u \in V_{i}} \sum_{v \in V_{+} \backslash V_{i}} y_{u} y_{v}=-\frac{1}{z_{i}^{2}} \sum_{j \leqslant a, j \neq i}\left\langle y^{i}, M y^{j}\right\rangle=-\frac{1}{z_{i}} \sum_{j \leqslant a, j \neq i} B_{i j} z_{j} .
$$

Analogous formula holds for $i>a$.

Let us start with some elementary properties of $B$. We have $\left\langle y^{i}, M y^{j}\right\rangle \leqslant 0$ if $i \neq j$ and $1 \leqslant i, j \leqslant a$, which implies that $B_{i j} \leqslant 0$ in this case. Similarly $B_{i j} \leqslant 0$ if $i \neq j$ and $a+1 \leqslant i, j \leqslant k+1$, and $B_{i j} \geqslant 0$ if $i \leqslant a<j$, or the other way around. Furthermore, we have $B z=0$; indeed,

$$
(B z)_{i}=\sum_{j=1}^{k+1} \frac{1}{z_{i}}\left\langle y^{i}, M y^{j}\right\rangle=\frac{1}{z_{i}}\left\langle y^{i}, M y\right\rangle=0 .
$$

Since the vectors $\widehat{y}^{i}(i=1, \ldots, k+1)$ are mutually orthogonal unit vectors, the matrix $B$ is a principal submatrix of $M$ in an appropriate orthonormal basis. By the Interlacing Eigenvalues Theorem, we have

$$
\lambda_{i}-\lambda_{k} \leqslant \mu_{i} \quad(i=1, \ldots, k+1) .
$$

Let $C$ be a symmetric $(k+1) \times(k+1)$ matrix, given by

$$
C_{i j}= \begin{cases}B_{i j} & \text { if } i \neq j \text { and either } i, j \leqslant a \text { or } i, j \geqslant a+1 \\ \Psi^{+}\left(V_{i}\right), & \text { if } i=j \leqslant a \\ \Psi^{-}\left(V_{i}\right), & \text { if } i=j \geqslant a+1 \\ 0, & \text { otherwise. }\end{cases}
$$

Then for $1 \leqslant i \leqslant a$, using (1),

$$
(C z)_{i}=\Psi^{+}\left(V_{i}\right) z_{i}+\sum_{j \leqslant a, j \neq i} B_{i j} z_{j}=0 .
$$


Similar computation works for $i>a$, to get $C z=0$.

Claim. The matrices $C$ and $C-B$ are positive semidefinite.

Indeed, from our discussion of the signs of the entries of $B$, it follows that the offdiagonal entries of $C$ and of $C-B$ are nonpositive. We also have $(C-B) z=C z=B z=0$, which implies that their diagonal entries are nonnegative. Let $D=\operatorname{diag}(z)$, then the matrix $D C D$ has nonnegative entries in the diagonal, nonpositive entries everywhere else, and every row-sum is 0 . So this matrix is diagonally dominant, and hence positive semidefinite, which implies that $C$ is positive semidefinite. For the matrix $C-B$ the conclusion follows similarly.

Next, we show that the largest eigenvalue of the matrix $C$ satisfies

$$
\lambda_{\max }(C)<2 c .
$$

Indeed, let $u$ be the eigenvector of $C$ belonging to $\lambda_{\max }(C)$. We may assume that $u_{1}=$ $z_{1}>0$ and $\left|u_{i}\right| \leqslant z_{i}$ for all $i$. Then, using that $C_{1 i} \leqslant 0$ for $i \neq 1$, we get

$$
\lambda_{\max }(C) u_{1}=\sum_{i} C_{1 i} u_{i} \leqslant C_{11} u_{1}-\sum_{i>1} C_{1 i} z_{i}=2 C_{11} u_{1}<2 c u_{1} .
$$

This proves (3).

Positive semidefiniteness of $C-B$ implies that $\mu_{k+1}$, the largest eigenvalue of $B$, is bounded above by $\lambda_{\max }(C)$. Hence by $(2)$,

$$
\lambda_{k+1}-\lambda_{k} \leqslant \mu_{k+1} \leqslant \lambda_{\max }(C)<2 c,
$$

which contradicts the choice of $c$.

The case $k=2$ is worth stating separately:

Corollary 3. If $y$ is an eigenvector belonging to $\lambda_{2}$, then both $G_{y}^{+}$and $G_{y}^{-}$are $\left(\lambda_{3}-\lambda_{2}\right) / 2$ expanders with respect to the weights $y_{i}^{2}$.

From our considerations, we can derive two other inequalities:

Corollary 4. If $y$ is an eigenvector belonging to $\lambda_{k}, a+b=k+1,\left\{U_{1}, \ldots, U_{a}\right\}$ is a partition of $\operatorname{supp}_{+}(y)$ and $\left\{V_{1}, \ldots, V_{b}\right\}$ is a partition of $\operatorname{supp}_{-}(y)$ with $w\left(U_{i}\right), w\left(V_{i}\right)>0$, then

$$
\lambda_{k+1}-\lambda_{k} \leqslant 2 \max \left\{\Psi^{+}\left(U_{1}\right), \ldots, \Psi^{+}\left(U_{a}\right), \Psi^{-}\left(V_{1}\right), \ldots, \Psi^{-}\left(V_{b}\right)\right\} .
$$

and

$$
\lambda_{k+1}-\lambda_{k} \leqslant \sum_{i=1}^{a} \Psi^{+}\left(U_{i}\right)+\sum_{i=1}^{b} \Psi^{-}\left(V_{i}\right) .
$$

(The second inequality is stronger in those cases only when the expansions of the sets are very different) 
Proof. The first inequality is an easy rephrasing of Theorem 2. To prove the second, it suffices to notice that the matrix $C$ in the proof above is positive semidefinite. Hence its largest eigenvalue is bounded above by its trace. Since $C-B$ is positive semidefinite, it follows that

$$
\mu_{a+b} \leqslant \operatorname{tr}(C)=\sum_{i} C_{i i}=\sum_{i=1}^{a} \Psi^{+}\left(U_{i}\right)+\sum_{i=1}^{b} \Psi^{-}\left(V_{i}\right) .
$$

\section{$3 \quad$ Examples}

There is no easy converse to Corollary 3. The following example shows that even if $\lambda_{2}=\lambda_{3}$, no separation property for the positive and negative supports of any eigenvector belonging to $\lambda_{2}$ follows.

Example 5. Let $C_{n}$ denote the cycle of length $n$, with its nodes labeled $0,1, \ldots, n-1$ in the natural order. Let $C_{n}^{k}$ denote the graph obtained from $C_{n}$ by connecting any two nodes at distance of at most $k$ along the cycle. The eigenvalues of the adjacency matrix are $\mu_{r}=2 \sum_{h=1}^{k} \cos (h r \pi i / n), r=0,1, \ldots n-1$. The smallest eigenvalue of the Laplacian is $\lambda_{1}=2-\mu_{0}=0$, and it is not hard to see that the second smallest eigenvalues are $\lambda_{2}=\lambda_{3}=2-\mu_{1}=2-\mu_{n-1}$. So the gap $\lambda_{3}-\lambda_{2}=0$. For every eigenvector $x$ belonging to $\lambda_{2}$, its positive support is induced by a half-cycle, which is easily seen to be a $c$-expander (even if weighted with the squared entries of the eigenvector) for $c=\Theta\left(k^{2} / n^{2}\right)$.

The assertion of the main theorem (or of its corollary) does not remain true without the weights, as the following example shows.

Example 6. Consider two isomorphic $D$-regular expanders $G_{1}$ and $G_{2}$ with $p$ nodes. Connect two corresponding nodes $a_{1}$ and $a_{2}$ in $G_{1}$ and $G_{2}$ by a path $P$ of length $q+1$, to get a connected graph $G$ with $n=2 p+q$ nodes. We assume that $q, p \rightarrow \infty$ and $q=o(p)$. Let $\lambda_{1}=0<\lambda_{2} \leqslant \ldots \leqslant \lambda_{n}$ be its eigenvalues of its Laplacian, with unit length eigenvectors $v_{1}=(1 / \sqrt{n}) \mathbb{1}, v_{2}, \ldots, v_{n}$.

It is not hard (but a little tedious) to see that this is a counterexample. Informally, the graph is "almost disconnected", and hence $\lambda_{2}$ is small (less than $2 / p$ ). On the other hand, $\lambda_{3}-\lambda_{2}$ will be of the same order as the eigenvalue gap of $G_{1}$, which is of constant magnitude. The positive support of $v_{2}$ will consist of $G_{1}$ and half of the path $P$, which is not an expander in the unweighted sense.

\section{References}

[1] N. Alon: Eigenvalues and expanders, Combinatorica 6(1986), 83-96.

[2] N. Alon and V.D. Milman: $\lambda_{1}$, isoperimetric inequalities for graphs and superconcentrators, J. Combinatorial Theory B 38(1985), 73-88.

[3] T. Biyıkoğlu, J. Leydold and P.F. Stadler: Laplacian Eigenvectors of Graphs, Lecture Notes in Math. 1915, Springer, 2007. 
[4] Y. Colin de Verdi'ere: Multiplicités des valeurs propres Laplaciens discrets et laplaciens continus, Rendiconti di Matematica 13 (1993), 433-460.

[5] Y. Colin de Verdière: Spectres de graphes, Cours Spécialisés 4, Société Mathématique de France, Paris, 1998.

[6] J. Dodziuk and W.S. Kendall: Combinatorial Laplacians and isoperimetric inequality, in: From Local Times to Global Geometry, Control and Physics, (ed. K. D. Ellworthy), Pitman Res. Notes in Math. Series 150 (1986), 68-74.

[7] E.B. Davies, G.M.L. Gladwell, J. Leydold and P.F. Stadler: Discrete nodal domain theorems, Linear Algebra and Apppl. 336 (2001), 51-60.

[8] A.M. Duval and V. Reiner: Perron-Frobenius type results and discrete versions of nodal domain theorems, Lin. Alg. Appl. 294 (1999), 259-268.

[9] M. Fiedler: A property of eigenvectors of nonnegative symmetric matrices and its application to graph theory, Czechoslov. Math. J. 25 (1975), 619-633.

[10] H. van der Holst: A short proof of the planarity characterization of Colin de Verdière, Journal of Combinatorial Theory, Series B 65 (1995) 269-272.

[11] H. van der Holst, L. Lovász, A. Schrijver: The Colin de Verdière graph parameter, in: Graph Theory and Combinatorial Biology, Bolyai Soc. Math. Stud. 7, János Bolyai Math. Soc., Budapest (1999), 29-85.

[12] M.R. Jerrum and A. Sinclair: Approximating the permanent, SIAM J. Comput. 18(1989), 1149-1178.

[13] Y. Lin, G. Lippner, D. Mangoubi and S.-T. Yau: Nodal geometry of graphs on surfaces, Discrete and Continuous Dynamical Systems 28 (2010), 1291-1298. 